\title{
Two Remarks on Kähler Homogeneous Manifolds
}

\author{
Bruce Gilligan $^{1}$ and Karl Oeljeklaus ${ }^{2}$
}

\begin{abstract}
We prove that every Kähler solvmanifold has a finite covering whose holomorphic reduction is a principal bundle. An example is given that illustrates the necessity, in general, of passing to a proper covering. We also answer a stronger version of a question posed by Akhiezer for homogeneous spaces of nonsolvable algebraic groups in the case where the isotropy has the property that its intersection with the radical is Zariski dense in the radical.

\section{Résumé}

Nous montrons que chaque variété homogène résoluble admet une revêtement finie dont la réduction holomorphe est un fibré principal. On donne un exemple qui montre qu'il est nécessaire en général de passer à une revêtement finie. Nous donnons de plus une réponse à une version plus forte d'une question d'Akhiezer pour un espace homogène d'un groupe algébrique non-résoluble dans le cas où l'isotropie est tel que l'intersection avec le radical est dense au sens de Zariski dans le radical.
\end{abstract}

\section{Introduction}

Let $G$ be a complex Lie group, $H \subset G$ a closed complex subgroup and $X:=$ $G / H$ the corresponding complex homogeneous manifold. One main question in the theory of complex homogeneous manifolds is to specify conditions on $G$ and $H$ under which the manifold $X$ admits a (not necessarily $G$-invariant) Kähler metric. For $X$ compact the theorem of Borel-Remmert (cf. [4]) gives a complete answer to the problem. In the non-compact case answers are

\footnotetext{
${ }^{1}$ Dept. of Mathematics and Statistics, University of Regina, Regina, Canada S4S 0A2 Email: gilligan@math.uregina.ca

${ }^{2}$ Centre de Mathématiques et d'Informatique, CNRS-UMR 6632 (LATP), 39, rue Joliot-Curie, Université de Provence, 13453 Marseille Cedex 13 France Email: Karl.Oeljeklaus@cmi.univ-mrs.fr
} 
known under certain assumptions on $G$, e.g. $G$ semisimple [3] or solvable [11]. In this paper we make two further contributions.

We first consider $X=G / H \rightarrow G / I=Y$ the holomorphic reduction of $X$, i.e., the unique $G$-equivariant fibration such that all holomorphic functions on $X$ come from the holomorphically separable base $Y$, see [5]. It has the universality property that every holomorphic map of $X$ into a holomorphically separable complex manifold factors through this fibration. Using known results, see [11], we prove that if $G$ is solvable and $X$ is Kähler then the holomorphic reduction of $X$ is, up to a finite covering, a principal bundle with a Cousin group as fiber. A Cousin group is a complex abelian Lie group admitting no non constant holomorphic functions.

Finally, we consider the following stronger version of a question posed by Akhiezer [1]: Suppose $G$ is a connected complex Lie group, $H$ a closed complex subgroup of $G$ and $X=G / H$ with $\mathcal{O}(X) \cong \mathbb{C}$ and there is no $G$ equivariant fibration of $X$ over a positive dimensional homogeneous rational manifold. Is it true that $X$ Kähler implies that $X$ is a Cousin group?

We prove that if $G$ is non-solvable algebraic and if the intersection of the radical $R \subset G$ with $H$ is Zariski dense in $R$ then $X$ is not Kähler.

\section{Kähler solvmanifolds}

Suppose $X=G / H$ is a Kähler solvmanifold, i.e., $G$ is a complex solvable Lie group. We consider its holomorphic reduction

$$
G / H \longrightarrow G / I
$$

and prove that it is always a principal bundle up to a finite covering. It is already known that its base $G / I$ is Stein [7] and that its fiber $C:=I / H$ is connected and is, as a complex manifold, biholomorphic to the Cousin group $I^{\circ} / H \cap I^{\circ}[11]$.

Theorem 1 Suppose $G$ is a connected, solvable, complex Lie group and $H$ is a closed complex subgroup of $G$ with $X:=G / H$ a Kähler manifold. Let

$$
G / H \longrightarrow G / I
$$

be the holomorphic reduction. Then there is a subgroup of finite index $\hat{I} \subset I$ such that the bundle

$$
\hat{X}:=G / \hat{I} \cap H \longrightarrow G / \hat{I}
$$


is a holomorphic $I^{\circ} / H \cap I^{\circ}$-principal bundle and represents the holomorphic reduction of $\hat{X}$.

Proof: Without loss of generality we may assume that $G$ is simply connected. Then $G$ admits a Hochschild-Mostow hull, see [6], i.e., there exists a solvable linear algebraic group

$$
G_{a}=\left(\mathbb{C}^{*}\right)^{k} \ltimes G
$$

and $G_{a}$ contains $G$ as a Zariski dense, topologically closed, normal complex subgroup.

Part I: We first assume that the isotropy is discrete. In order to underline this fact, we will write the isotropy subgroup as $\Gamma$ in this part of the proof. Let

$$
X=G / \Gamma \longrightarrow G / J \cdot \Gamma
$$

be the holomorphic reduction of $X$ with $J$ connected. Because $X$ is Kähler, the fiber $J \cdot \Gamma / \Gamma$ of its holomorphic reduction is (as a complex manifold) isomorphic to a Cousin group [11]. We remark now that $G_{a} / \Gamma$ is biholomorphic to $\left(\mathbb{C}^{*}\right)^{k} \times(G / \Gamma)$. As a consequence, $G_{a} / \Gamma$ is Kähler and its holomorphic reduction is given by

$$
G_{a} / \Gamma \longrightarrow G_{a} / J \cdot \Gamma \cong\left(\mathbb{C}^{*}\right)^{k} \times(G / J \cdot \Gamma),
$$

because the base of this fibration is holomorphically separable and its fiber is $J \cdot \Gamma / \Gamma$ and is, as a complex manifold, biholomorphic to a Cousin group. Let $G_{a}(\Gamma)$ denote the algebraic closure of $\Gamma$ in $G_{a}$. The manifold $G_{a} / G_{a}(\Gamma)$ is Stein, as a quotient of algebraic solvable Lie groups. In view of the universality property of the holomorphic reduction one has the following commutative diagram

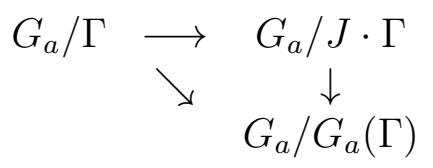

In particular, $J \cdot \Gamma \subset G_{a}(\Gamma)$.

Now consider the fibration

$$
G_{a}(\Gamma) / \Gamma \longrightarrow G_{a}(\Gamma) / J \cdot \Gamma .
$$

This fibration is the holomorphic reduction of $G_{a}(\Gamma) / \Gamma$, because its fiber is, as a complex manifold a Cousin group, and its base, as an orbit in $G_{a} / J \cdot \Gamma$, 
is holomorphically separable and hence Stein.

Let $G_{a}(\Gamma)^{\circ}$ be the connected component of the identity of $G_{a}(\Gamma)$ and define $\hat{\Gamma}:=\Gamma \cap G_{a}(\Gamma)^{\circ}$. Then $\hat{\Gamma}$ is a Zariski dense subgroup of $G_{a}(\Gamma)^{\circ}$. Because the quotient $G_{a}(\Gamma)^{\circ} / \hat{\Gamma}$ is Kähler and $\hat{\Gamma}$ is Zariski dense in $G_{a}(\Gamma)^{\circ}$, it follows that $G_{a}(\Gamma)^{\circ}$ is a unipotent, see Lemma 3, p. 412 in [11].

Therefore

$$
G_{a}(\Gamma)^{\circ} / \hat{\Gamma} \longrightarrow G_{a}(\Gamma)^{\circ} / J \cdot \hat{\Gamma}
$$

is the holomorphic reduction of the homogeneous Kähler nilmanifold $G_{a}(\Gamma)^{\circ} / \hat{\Gamma}$ the fiber of which is still biholomorphic to $J / \Gamma \cap J$.

Let us introduce the following notation: Let $\mathfrak{g}_{a}$ denote the Lie algebra of the connected unipotent group $G_{a}(\Gamma)^{\circ}$, and $Z$ denote its center, with its Lie algebra being $\mathfrak{z}$. Since the exponential map exp $: \mathfrak{g} \rightarrow G_{a}(\Gamma)^{\circ}$ is bijective we may consider the real Lie algebra $\mathfrak{g}_{\mathbb{R}}$ spanned by $\exp ^{-1}(\hat{\Gamma})$ and its maximal complex ideal $\mathfrak{m}:=\mathfrak{g}_{\mathbb{R}} \cap i \mathfrak{g}_{\mathbb{R}}$.

The construction of the holomorphic reduction of a complex nilmanifold given in [5] shows that the group $J$ is the smallest connected, closed, complex subgroup of $G_{a}(\Gamma)^{\circ}$ that contains the connected group corresponding to the ideal $\mathfrak{m}$ and such that $J \cdot \hat{\Gamma}$ is closed in $G_{a}(\Gamma)^{\circ}$. We note that $Z$ is a group such that $Z \cdot \hat{\Gamma}$ is closed, as follows from a result of Barth-Otte; see Theorem 4 in [5]. By using the Kähler assumption and detailed Lie algebra calculations it is shown in the proof of Theorem 2', p. 409 in [11] that

$$
\mathfrak{m} \subset \mathfrak{z} .
$$

As a consequence, $J \subset Z$. In particular, $J$ centralizes $\hat{\Gamma}$ in the nilpotent group $G_{a}(\Gamma)^{\circ}$. Hence $\hat{\Gamma} \triangleleft J \cdot \hat{\Gamma}$ and thus $J \cdot \hat{\Gamma} / \hat{\Gamma}$ is a group. We note also that $\hat{I}:=J \cdot \hat{\Gamma}$ is a subgroup of finite index of $J \cdot \Gamma$ and $\hat{I} \cap \Gamma=\hat{\Gamma}$. Thus the holomorphic reduction $(\dagger)$ is a $J \cdot \hat{\Gamma} / \hat{\Gamma}=\hat{I} / \hat{\Gamma}$-principal bundle and the theorem is proved in this case.

Part II: We now assume that the isotropy subgroup $H$ is not discrete in $G$. Let

$$
G / H \longrightarrow G / I
$$

be the holomorphic reduction of $G / H$. We would like to show that $H$ is a normal subgroup of $I$. Here again $G_{a} / H$ is biholomorphic to $\left(\mathbb{C}^{*}\right)^{k} \times G / H$ and is therefore Kähler. Also $G_{a} / H \rightarrow G_{a} / I$ is clearly the holomorphic 
reduction of $G_{a} / H$. Set $N:=\left(N_{G_{a}}\left(H^{\circ}\right)\right)^{H}$, i.e., those connected components of the normalizer of $H^{\circ}$ in $G_{a}$ that meet $H$. Note that $N / H$ is connected and $N$ is an algebraic subgroup of $G_{a}$. Therefore, $N^{\circ}$ has finite index in $N$. We remark that $G_{a} / N$ is holomorphically separable by Lie's Flag Theorem. Again by the universality property of the holomorphic reduction one has the following diagram

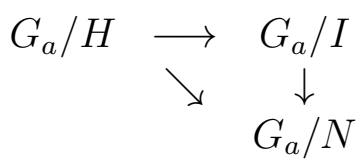

In particular, $I \subset N$. Since $N / I$ is an orbit in $G_{a} / I$ and the latter space is Stein, it follows that $N / I$ is holomorphically separable. But we are in a Kähler setting and thus the fiber $I / H$ of the holomorphic reduction of $G / H$ is biholomorphic to a (connected) Cousin group. As a consequence, $N / H \rightarrow N / I$ is the holomorphic reduction of $N / H$. Now define $\hat{I}:=I \cap N^{\circ}$. Then $\hat{I} \cap H=N^{\circ} \cap H$. Moreover, $\hat{I} \subset G$ and $\hat{I} \cap H \subset G$. With this notation we get as in the first case the fibration

$$
N / H \simeq N^{\circ} /\left(N^{\circ} \cap H\right) \rightarrow N^{\circ} / \hat{I} \simeq N / I
$$

which is the holomorphic reduction of $N^{\circ} /\left(N^{\circ} \cap H\right)$ with fiber $\hat{I} /\left(N^{\circ} \cap H\right)$.

Now we have the following

$$
\left(N^{\circ} / H^{\circ}\right) /\left(N^{\circ} \cap H / H^{\circ}\right) \simeq N^{\circ} / N^{\circ} \cap H \longrightarrow N^{\circ} / \hat{I} \simeq\left(N^{\circ} / H^{\circ}\right) /\left(\hat{I} / H^{\circ}\right)
$$

and Part I above applies. It follows that $N^{\circ} \cap H / H^{\circ}$ is a normal subgroup of $\left(N^{\circ} \cap H / H^{\circ}\right) \cdot\left(I^{\circ} / H^{\circ}\right)$ and that $N^{\circ} \cap H$ is a normal subgroup of $\hat{I}$ which in turn is a subgroup of finite index of $I$. Thus the holomorphic reduction of the Kähler solvmanifold $G / H$ is, up to a finite covering, a Cousin group principal bundle.

The following examples show that, in general, passing to a subgroup of finite index is unavoidable in order to get a principal bundle and that the Kähler condition is necessary.

Example 1: Let

$$
G:=\left\{\left(\begin{array}{ll}
a & b \\
0 & 1
\end{array}\right) \mid a \in \mathbb{C}^{*}, b \in \mathbb{C}\right\}
$$


and

$$
\Gamma:=\left\{\left(\begin{array}{cc}
\sqrt{-1}^{n} & k+\sqrt{-1} l \\
0 & 1
\end{array}\right) \mid n, k, l \in \mathbb{Z}\right\} .
$$

Then $X:=G / \Gamma$ is Kähler, $\Gamma$ is not nilpotent, and the holomorphic reduction is not a principal bundle. But of course all this is true, if one replaces $\Gamma$ by the obvious subgroup of index 4 .

Example 2: We also note that the Kähler condition is necessary. We give the following example due to J.J. Loeb [8]. Let $K=\mathbb{Z}, \mathbb{C}$ and $G_{K}=K \ltimes K^{2}$ be defined by:

$$
(z, b) \circ\left(z^{\prime}, b^{\prime}\right):=\left(z+z^{\prime}, e^{A z^{\prime}} b+b^{\prime}\right),
$$

where $z, z^{\prime} \in K, \quad b, b^{\prime} \in K^{2}$ and $A$ is the real logarithm of the matrix $\left(\begin{array}{ll}2 & 1 \\ 1 & 1\end{array}\right)$. The complex solvmanifold $X=G_{\mathbb{C}} / G_{\mathbb{Z}}$ is not Kähler and has as holomorphic reduction

$$
G_{\mathbb{C}} / G_{\mathbb{Z}} \stackrel{\mathbb{C}^{*} \times \mathbb{C}^{*}}{\longrightarrow} G_{\mathbb{C}} / G_{\mathbb{C}}^{\prime} \cdot G_{\mathbb{Z}}=\mathbb{C}^{*}
$$

No finite covering of $X=G_{\mathbb{C}} / G_{\mathbb{Z}}$ has a holomorphic reduction that is a principal bundle since in this case $X$ would be Stein and thus Kähler.

\section{A non-Kähler criterion}

For a complex Lie group $G$ we let $G=S \cdot R$ be a Levi-Malcev decomposition, where $S$ is semisimple and $R$ is the radical of $G$.

Theorem 2 Suppose $G=S \cdot R$ is a Levi-Malcev decomposition of an algebraic group with both factors having positive dimension. Let $H$ be a closed complex subgroup of $G$ and $X:=G / H$ such that $\mathcal{O}(X) \cong \mathbb{C}$. Assume $H$ is not contained in any proper parabolic subgroup of $G$, i.e. there is no $G$ equivariant fibration of $X$ over a positive dimensional homogeneous rational manifold. Suppose $R \cap H$ is Zariski dense in $R$. Then $G / H$ is not Kähler.

Proof: Without loss of generality we may assume that the $G$-action on $X$ is almost effective. Let $N:=N_{G}\left(H^{\circ}\right)$ be the normalizer of the identity component of $H$ in $G$. Then $G / N$ is an orbit in some projective space and our assumptions force $N=G$, i.e. $H$ is discrete. We shall use $\Gamma$ instead of $H$ for this reason. We assume that $X$ is Kähler and derive a contradiction. 
It is known that the orbits of $R$ are closed in our situation, see [12], and so we may consider the fibration

$$
G / \Gamma \longrightarrow G / R \cdot \Gamma=S /(S \cap R \cdot \Gamma) .
$$

Note that $\Lambda:=S \cap R \cdot \Gamma$ is a discrete subgroup of $S$. It follows from the assumptions on $X$ that $\Lambda$ is a Zariski dense subgroup of $S$, since every algebraic subgroup of a semisimple complex Lie group is contained in either a maximal reductive or a maximal parabolic subgroup of $G$. Recall that a homogeneous manifold of a semisimple Lie group is Stein if and only if the isotropy group is reductive, [9], [10].

As a consequence, we can find a semisimple element $\lambda \in \Lambda$ of infinite order. The Zariski closure of the infinite cyclic subgroup generated by $\lambda$ is an algebraic torus $A:=\overline{\langle\lambda\rangle_{\mathbb{Z}}} \cong\left(\mathbb{C}^{*}\right)^{l}$ for some $l>0$. Without loss of generality, we may assume $A$ is connected, since one can replace $\lambda$ by a finite power, if necessary. Note that $A /\langle\lambda\rangle$ is a Cousin group and therefore $A / A \cap \Lambda$ is also Cousin. Now let $S=S_{1} \cdots S_{n}$ be a decomposition of $S$ into a product of simple factors and let $p_{j}: S \rightarrow S_{j}$ denote the projection onto the $j$-th factor $S_{j}$. It was noted in the Lemma on p. 328 in [2] that one may choose $\lambda$ so that

$$
p_{j}(A) \neq\{e\} \text { for all } j=1, \ldots, n \text {. }
$$

Set $G^{*}:=A \ltimes R$ which is an algebraic solvable subgroup of $G$ and $\Gamma^{*}:=$ $G^{*} \cap \Gamma$. Let $N$ be the Zariski closure of $\Gamma^{*}$ in $G^{*}$. Then $N \subset G^{*}$ and $N^{\circ}$ is nilpotent by Lemma 4.3, p. 412, in [11].

We claim $N=G^{*}$. Consider the fibration $\pi: G^{*} / \Gamma^{*} \rightarrow A / A \cap \Lambda$ and its restriction to the $N^{\circ}$-orbit $N^{\circ} / \Gamma^{*} \cap N^{\circ}$. The fiber of the restriction $\left.\pi\right|_{N^{\circ} / \Gamma^{*} \cap N^{\circ}}$ is equal to $R /(R \cap \Gamma)$ by our assumption that $\Gamma \cap R$ is Zariski dense in $R$. This implies $R \subset N^{\circ}$. Now the projection $p: G \rightarrow S \cong G / R$ is an algebraic homomorphism and thus the image of an algebraic subgroup of $G$ containing $R$ is an algebraic subgroup of $S$. Hence $p\left(N^{\circ}\right)$ is an algebraic subgroup of $S$ that contains $\langle\lambda\rangle_{\mathbb{Z}}$. As a consequence, $p\left(N^{\circ}\right)=A$. These two observations imply $N^{\circ}=G^{*}$. Thus $G^{*}$ is nilpotent.

Let $\phi$ denote the representation of $A$ on $R$ in the semidirect product $G^{*}=A \ltimes_{\phi} R$. Since $A \cong\left(\mathbb{C}^{*}\right)^{l}$, it follows that $\phi(A)$ is reductive. But $\phi(A)$ is also nilpotent and so must be trivial. Thus $A$ acts trivially on the radical $R$. Therefore $G^{*}=A \times R$ is a direct product. But this implies that the 
group $G$ is a group theoretic direct product $S \times R$ by the choice of $A$, see $(*)$, and the fact, just proved, that the subgroup $A$ is contained in the kernel of the representation that builds the semidirect product $S \ltimes R$. This yields the desired contradiction, since $S$ cannot have positive dimension in the case of a Kähler quotient of a direct product $S \times R$, see [12].

Acknowledgements: The first author gratefully acknowledges that he was partially supported by an NSERC Discovery Grant and was an Invité du LATP à l'Université de Provence.

\section{References}

[1] D. AkHiezer, Invariant analytic hypersurfaces in complex nilpotent Lie groups, Ann. Global Anal. Geom., 2 (1984), 129-140.

[2] D. AkHIEzER, Invariant meromorphic functions on complex semisimple Lie groups, Invent. Math. 65 (1982), 325 - 329.

[3] F. Berteloot, Existence d'une structure kählérienne sur les variétés homogènes semi-simples, C.R. Acad. Sci. Paris, Sér. I, 305 (1987), 809812 .

[4] A. Borel And R. Remmert, Über kompakte homogene Kählersche Mannigfaltigkeiten, Math. Ann. 145 (1962), 429-439.

[5] B. Gilligan and A. T. Huckleberry, On Non-Compact Complex Nil-Manifolds, Math. Ann. 238 (1978), 39 - 49.

[6] G. Hochschild and G.D. Mostow, On the algebra of representative functions of an analytic group. II, Amer. J. Math., 86 (1964), 869-887.

[7] A. Huckleberry and E. Oeljeklaus, On holomorphically separable complex solvmanifolds, Ann. Inst. Fourier (Grenoble) 36 (1986), 57-65.

[8] J-J LoEB, Fonctions plurisousharmoniques sur un groupe de Lie complexe invariantes par une forme réelle, C.R. Acad. Sci. Paris, Sér. I, 299 (1984), 663-666. 
[9] Y. Matsushima, Espaces homogènes de Stein des groupes de Lie complexes, Nagoya Math. J. 16 (1960), 205-218.

[10] A. L. Onishchik, Complex envelopes of compact homogeneous spaces, Dokl. Acad. Nauk SSSR 130 (1960), 726-729.

[11] K. Oelueklaus and W. Richthofer, On the Structure of Complex Solvmanifolds, J. Diff. Geom. 27 (1988), 399 - 421.

[12] K. Oeljeklaus and W. Richthofer, Recent results on homogeneous complex manifolds. Complex Analysis III, (College Park, Md., 1985 - 86), 78 - 119, Lecture Notes in Math. 1277, Springer, Berlin, 1987. 